07

\title{
Упрощенная модель для расчета мощностных и спектральных характеристик лазерного диода с волоконной брэгговской решеткой
}

\author{
(C) В.Д. Курносов, К.В. Курносов \\ Научно-исследовательский институт „Полюс“ им. М.Ф. Стельмаха, \\ 117342 Москва, Россия \\ e-mail: webeks@mail.ru
}

(Поступило в Редакцию 9 июля 20015 г. В окончательной редакции 23 мая 2016 г.)

По упрощенной модели, которая включает в себя лазерный диод, воздушный зазор и световод, проведены расчеты мощностных и спектральных характеристик, а также ширины линии генерации лазера с волоконной брэгговской решеткой. Показано, что упрощенная модель лазера дает удовлетворительное совпадение теории и эксперимента для мощностных и спектральных характеристик. Показано, что величина ширины линии генерации для лазерного диода с волоконной брэгговской решеткой может быть уменьшена на четыре порядка по сравнению с шириной линии генерации для одиночного лазерного диода.

DOI: 10.21883/JTF.2017.03.44251.1506

\section{Введение}

В настоящее время исследованию моделей лазерного диода (ЛД) с внешним резонатором, включая резонаторы с волоконной брэгговской решеткой (ВБР), посвящено большое число работ, начиная с модели, представленной в работе [1], и заканчивая рядом работ, направленных на улучшение этой модели [2-4].

Недостатком всех моделей, рассмотренных в работах $[1-4]$, является то, что не учитывается распределение оптических полей внутри такой сложной системы, которой является лазерный диод с брэгговской решеткой в волокне. Поэтому в работе [5] за основу была взята модель, рассмотренная в работе [6]. Показано, что в данной модели можно получить режим одночастотной генерации. В работе [7] с учетом давления, возникающего после напайки брэгговской решетки на термоохладитель, дисперсии, а также температуры окружающей среды, определены области изменения тока, при которых возможна настройка лазера на $D_{2}$-линию цезия. Однако в этой работе не учитывался нагрев ЛД за счет протекающего тока накачки.

В работе [8] проведены расчеты пороговых, мощностных и спектральных характеристик ЛД с ВБР. Исследовано влияние на эти характеристики нагрева активной области лазерного диода, его теплового сопротивления и величины мощности излучения, выводимой из резонатора излучателя. Показано, что удовлетворительное совпадение теории и эксперимента наблюдается в случае учета нагрева активной области лазерного диода протекающим через него током накачки и мощности излучения, выводимой из резонатора излучателя.

В работе [9] исследуются мощностные и спектральные характеристики ЛД с ВБР с различной длиной световода. Получено упрощенное выражение для коэффициента усиления лазерного диода.

Основной интерес разработчиков систем состоит в том, что спектр излучения ЛД с селективным внешним резонатором становится одночастотным и уменьшается ширина линии генерации (ШЛГ) ЛД. В работе [10] получено выражение (25), позволяющее провести расчет ШЛГ с учетом внешней пассивной области. Для этого в настоящей работе проведено упрощение модели ЛД с ВБР, рассмотренной в работе [5], чтобы иметь трех-, а не четырехкомпонентную систему. Показано удовлетворительное совпадение с экспериментом спектральных и ватт-амперных характеристик (ВтАХ). Проведен расчет ШЛГ и показано, что величина ширины линии генерации для ЛД с ВБР может быть уменьшена на четыре порядка по сравнению с шириной линии генерации одиночного ЛД.

\section{1. Модель}

На рис. 1 представлен упрощенный по сравнению с моделью, рассмотренной в работе [5], вариант лазера, который включает в себя ЛД, воздушный зазор и световод. ВБР моделируется коэффициентом отражения, зависящим от длины волны излучения. Из рассмотрения исключена зависимость длины резонатора лазера от температуры, и использовано упрощенное выражение для коэффициента усиления лазерного диода.

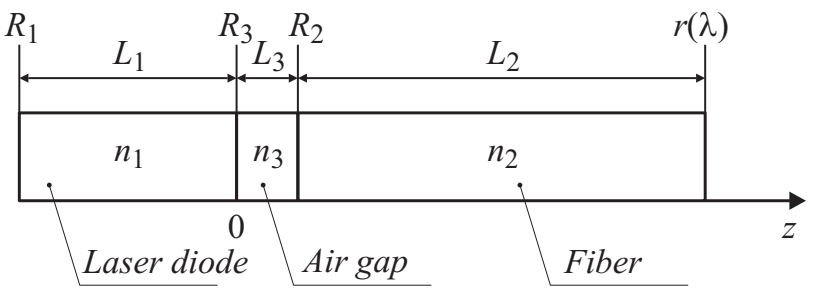

Рис. 1. Схема упрощенной модели полупроводникового лазера: $L_{1}, n_{1}, L_{2}, n_{2}, L_{3}, n_{3}$ - длины и показатели преломления полупроводникового лазера, волоконного световода и воздушного зазора, $R_{1}, R_{2}, R_{3}$ - коэффициенты отражения, $r(\lambda)$ - коэффициент отражения брэгговской решетки. 
Поле внутри резонатора представим следующим образом [6]:

$$
U_{i}= \begin{cases}A_{i} \sin \left[\beta_{1 i}\left(z+L_{1}\right)\right] & -L_{1} \leq z \leq 0, \\ B_{i} \sin \left(\beta_{3 i} z\right)+C_{i} \cos \left(\beta_{3 i} z\right) & 0 \leq z \leq L_{3}, \\ D_{i} \sin \left[\beta_{2 i}\left(z-\left(L_{2}+L_{3}\right)\right)\right] & L_{3} \leq z \leq L_{2}+L_{3},\end{cases}
$$

где индекс $i$ обозначает моду резонатора с длиной волны $\lambda_{i}, \beta_{1 i}=2 \pi n_{1} / \lambda_{i}, \beta_{2 i}=2 \pi n_{2} / \lambda_{i}, \beta_{3 i}=2 \pi n_{3} / \lambda_{i}-$ постоянные распространения в соответствующих областях. В (1) автоматически выполняются граничные условия $U_{i}\left(-L_{1}\right)=U_{i}\left(L_{2}+L_{3}\right)=0$.

Сшивая решения для поля $U_{i}(z)$ и производной $d U_{i}(z) / d z$ в точках $z=0, L_{3}$, получаем характеристическое уравнение, определяющее длины волн излучения, которое может распространяться в системе, показанной на рис. 1:

$$
a_{11 i} d_{11 i}-a_{12 i} d_{12 i}=0,
$$

где

$$
\begin{gathered}
a_{11 i}=\beta_{3 i} \sin \left(\beta_{3 i} L_{3}\right) \sin \left(\beta_{2 i} L_{2}\right)-\beta_{2 i} \cos \left(\beta_{3 i} L_{3}\right) \cos \left(\beta_{2 i} L_{2}\right), \\
a_{12 i}=\beta_{2 i} \sin \left(\beta_{3 i} L_{3}\right) \cos \left(\beta_{2 i} L_{2}\right)+\beta_{3 i} \cos \left(\beta_{3 i} L_{3}\right) \sin \left(\beta_{2 i} L_{2}\right), \\
d_{12 i}=\beta_{1 i} \cos \left(\beta_{1 i} L_{1}\right), \quad d_{11 i}=\beta_{3 i} \sin \left(\beta_{1 i} L_{1}\right) .
\end{gathered}
$$

Коэффициент отражения, расположенный на правой стороне световода (рис. $1, z=L_{2}+L_{3}$ ), рассчитывается по формуле

$$
r=\frac{\gamma_{1 i} \cosh \left(\gamma_{i}-L_{B}\right)-\chi_{i} \sinh \left(\gamma_{i} L_{B}\right)}{\gamma_{1 i} \cosh \left(\gamma_{i} L_{B}\right)+\theta_{i} \sinh \left[\gamma_{i} L_{B}\right]} .
$$

Коэффициенты, входящие в (4), определяются следующим образом: $\gamma_{i}^{2}=\left(\alpha_{B} / 2+j \delta_{i}\right)^{2}+K_{0}^{2}, \delta_{i}=\beta_{i}-\beta_{0}=$ $=2 \pi n_{B}\left(\lambda_{i}^{-1}-\lambda_{B}^{-1}\right), \gamma_{1 i}=\xi \gamma_{i}, \xi=r_{0} \exp \left(-j 2 \beta_{0} L_{B}\right), r_{0}=$ $=\sqrt{R_{B}}, \theta_{i}=\left(\alpha_{B} / 2+j \delta_{i}\right)+J K_{0} \xi, \chi_{i}=\left(\alpha_{B} / 2+j \delta_{i}\right) \xi+j K_{0}$, где $\gamma_{i}$ - дисперсионное соотношение, $\alpha_{B}$ - потери в брэгговской решетке, $K_{0}$ - коэффициент связи между встречными волнами. Коэффициент отражения по мощности равен $R=|r|^{2}$.

Плотность фотонов в $i$-ой моде резонатора ЛД равна

$$
S_{1 i}=\frac{\beta F_{1 i} R_{\mathrm{sp}}}{\tau_{i}^{-1}-F_{1 i} G_{i}}
$$

где $\beta$ - коэффициент, учитывающий вклад спонтанного излучения в генерирующую моду, остальные обозначения даны ниже.

Время жизни фотона в резонаторе равно

$$
\tau_{i}=\left[c_{0}\left(\frac{F_{1 i}}{n_{1 i}} \alpha_{1 \Sigma}+\frac{F_{2 i}}{n_{2}} \alpha_{2 \Sigma}+\frac{F_{3 i}}{n_{3}} \alpha_{3 \Sigma}\right)\right]^{-1} .
$$

Коэффициенты $F_{i}$, входящие в (5) и (6), определяются как

$$
F_{1 i}=\left[1+\left(\frac{n_{2}}{n_{1 i}}\right)^{2} \frac{V_{2}}{V_{1}} d_{13 i}^{2}\right]^{-1},
$$

$$
\begin{gathered}
F_{2 i}=\left[1+\left(\frac{n_{1 i}}{n_{2}}\right)^{2} \frac{V_{1}}{V_{2}} d_{13 i}^{-2}\right]^{-1}, \\
F_{3 i}=\frac{d_{11 i}^{2}+d_{12 i}^{2}}{\beta_{3 i}}\left[\left(\frac{n_{1}}{n_{3}}\right)^{2} \frac{V_{1}}{V_{3}}+\left(\frac{n_{2}}{n_{3}}\right)^{2} \frac{V_{2}}{V_{3}} d_{13 i}^{2}\right]^{-1},
\end{gathered}
$$

где $d_{13 i}=-\left[d_{11 i} \cos \left(\beta_{3 i} L_{3}\right)+d_{12} \sin \left(\beta_{3 i} L_{3}\right)\right] /\left[\beta_{3 i} \sin \left(\beta_{2 i} L_{2}\right)\right]$, a $d_{11 i}$ и $d_{12 i}$ определяются формулами (3), $V_{1}, V_{2}, V_{3}-$ объемы соответствующих областей (см. рис. 1).

Оптические потери $\alpha_{i}$ равны

$$
\begin{gathered}
\alpha_{1 \Sigma}=\alpha_{10}+\frac{1}{2 L_{1}} \ln \frac{1}{R_{i}}, \\
\alpha_{2 \Sigma}=\alpha_{20}+\frac{1}{2 L_{2}} \ln \frac{1}{\left(1-R_{2}\right)^{2}}+\frac{1}{2 L_{2}} \ln \frac{1}{|r(\lambda)|^{2}}, \\
\alpha_{3 \Sigma}=\alpha_{30}+\frac{1}{2 L_{3}} \ln \frac{1}{\left(1-R_{3}\right)^{2}}, \\
\alpha_{30}=\frac{1}{L_{3}} \ln \frac{1}{k_{\text {in }}},
\end{gathered}
$$

где $k_{\text {in }}$ - эффективность ввода излучения из лазера в волоконный световод.

Необходимо отметить, что потери в ВБР включены в состав потерь в световоде в виде распределенных потерь $\left(2 L_{2}\right)^{-1} \ln \left(\left|r(\lambda)^{-2}\right|\right)$.

Коэффициент усиления зависит от плотности носителей в активной области лазера

$$
G_{i}=Q_{i}\left(n_{a} D_{i}-n_{a 0}\right),
$$

где $Q_{i}=\left(c_{0} \Gamma_{a} / n_{1 i}\right)\left(d g / d n_{a}\right), D_{i}=1-\left[2\left(E_{i}-E_{g}\right) / \Delta E_{g}\right]^{2}$, $E_{i}=1.24 / \lambda_{i}, \lambda_{i}$ - длина волны излучения $i$-й моды в микрометрах, $d g / d n_{a}$ - дифференциальный коэффициент усиления, $\Delta E_{g}=0.18 \mathrm{eV}$ - ширина спектра коэффициента усиления, $n_{a 0}=1.75 \cdot 10^{18} \mathrm{~cm}^{-3}-$ плотность носителей, при которой коэффициент усиления равен нулю.

Для величины $E_{g}$ получено следующее выражение:

$$
E_{g}=E_{0}-5.4 \cdot 10^{-4} \frac{T^{2}}{204+T}-2 k_{g} n_{a}^{1 / 3},
$$

где $E_{0}=1.63 \mathrm{eV}$.

Температуру активной области ЛД запишем в виде

$$
T=T_{0}+\delta T
$$

Нагрев активной области за счет протекающего через ЛД тока накачки $I$ и плотности фотонов, выводимых из резонатора ЛД, представим в виде [8]

$$
\delta T=R_{T}\left(U_{p n} I+I^{2} R_{g}-2 P_{1}\right),
$$

где $R_{T}$ - тепловое сопротивление ЛД, $U_{p n}-$ напряжение на $p-n$-переходе, $R_{g}$ - динамическое сопротивление ЛД. 
Скорость спонтанной рекомбинации носителей равна

$$
R_{\mathrm{sp}}=B n_{a}^{2}
$$

где $B-$ коэффициент, который считается постоянной величиной.

Оптическая мощность на выходе резонатора ЛД с коэффициентом отражения $R_{1}$ равна

$$
P_{1}=h v \frac{c_{0}}{n_{1 \mathrm{gr}}} A_{c}\left(1-R_{1}\right) \sum_{i} S_{1 i},
$$

где $A_{c}$ - площадь поперечного сечения излучающей области лазера. Оптическая мощность на выходе объектива излучателя принимается равной $P=0.8 P_{1}$.

Ток накачки излучателя

$$
I=I_{\mathrm{th}}+q V_{a} \frac{c_{0}}{n_{\mathrm{gr}}} \sum_{i} \Gamma_{a} g_{i} S_{1 i}
$$

где $V_{a}$ - объем активной области ЛД. Величина порогового тока $I_{\mathrm{th}}$ включает в себя излучательную и безызлучательную скорости рекомбинации носителей:

$$
I_{\mathrm{th}}=q V_{a}\left(R_{\mathrm{sp}}+A n_{a}\right),
$$

где $A-$ коэффициент безызлучательной рекомбинации, $n_{a}-$ плотность носителей в активной области ЛД.

Показатель преломления ЛД может быть представлен как

$$
\begin{aligned}
n_{1}\left(\lambda, T_{\mathrm{LD}}\right)= & n_{10}\left\{1+\frac{1}{n_{10}} \frac{\partial n_{1}}{\partial \lambda}\left(\lambda-\lambda_{B}\right)\right. \\
& \left.+\frac{1}{n_{10}}\left(\frac{\partial n_{1}}{\partial T_{\mathrm{LD}}}\right)\left(T_{\mathrm{Ld}}-T_{0}\right)\right\},
\end{aligned}
$$

где $n_{10}-$ показатель преломления ЛД при $T_{\mathrm{LD}}=T_{0}$ и $\lambda=\lambda_{B}, \lambda_{B}$ - длина волны Брэгга, $T_{\mathrm{LD}}-$ температура активной области ЛД, $T_{0}$ - температура окружающей среды, $\partial n_{1} / \partial T_{\mathrm{LD}}-$ изменение показателя преломления с изменением температуры активной области ЛД.

В работе [10] показано, что ШЛГ ЛД с ВБР может быть получена умножением ШЛГ одиночного ЛД $\Delta f_{\mathrm{LD}}$ на величину $F_{1}^{2}$

$$
\Delta f_{\mathrm{LD}+\mathrm{FBG}}=\Delta f_{\mathrm{LD}} F_{1}^{2} .
$$

Ширина линии генерации одиночного ЛД получена из формулы (26) работы [11] заменой величины $P_{0}$ на $P_{0}=\hbar \omega \alpha\left(I-I_{\text {th }}\right) /\left[q\left(\alpha_{\text {end }}+\alpha_{10}\right)\right]:$

$$
\Delta f_{\mathrm{LD}}=\frac{q n_{\mathrm{sp}}\left(1+\alpha_{H}^{2}\right)\left(\alpha_{01}+\alpha_{\mathrm{end}}\right)^{2} v_{\mathrm{gr}}^{2}}{8 \pi\left(I-I_{\mathrm{th}}\right)},
$$

где $v_{\mathrm{gr}}$ - групповая скорость света. Величина $F_{1}$ для $i$-й моды резонатора определяется формулой (7) настоящей работы.
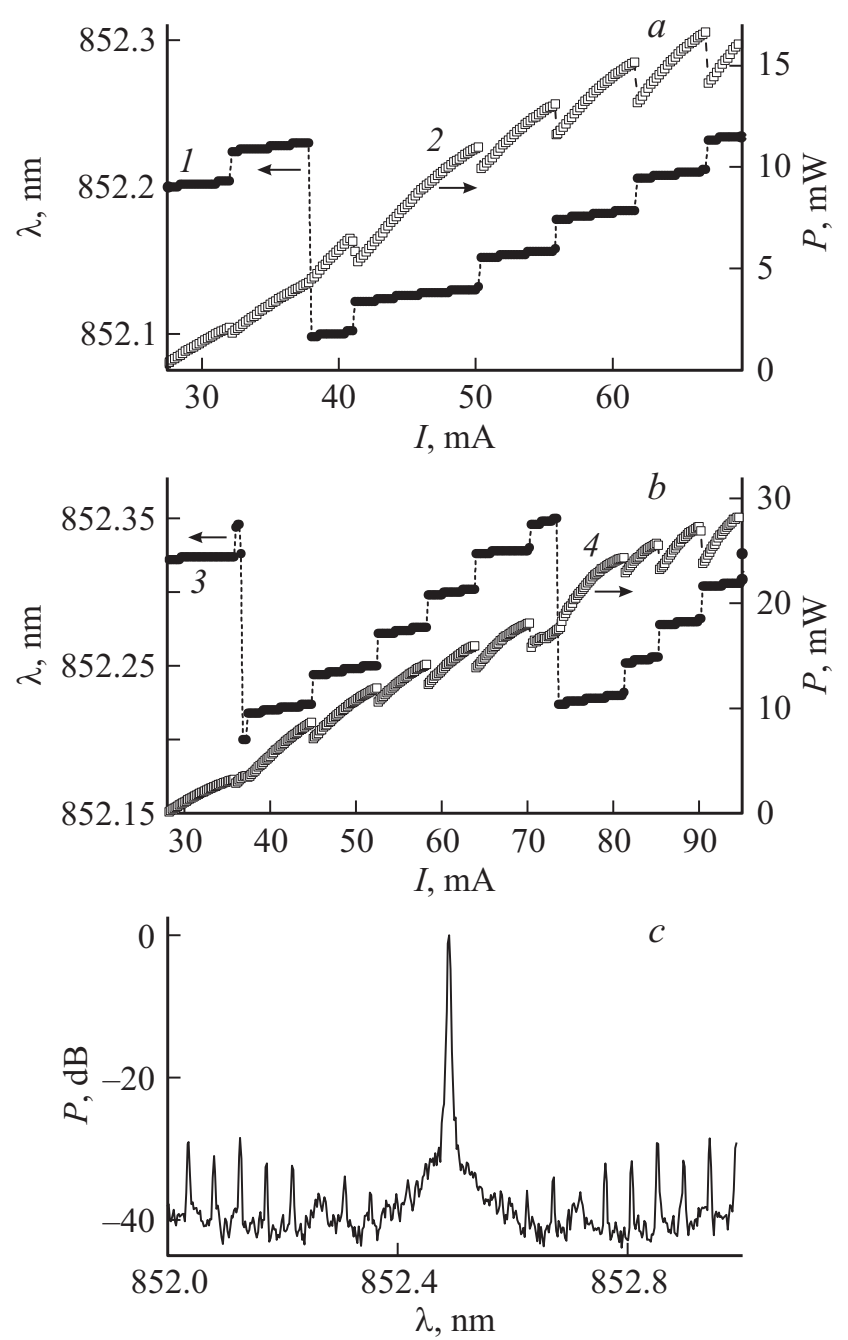

Рис. 2. Экспериментальные зависимости длины волны $(1,3)$, мощности $(2,4)$ и спектра излучения от величины тока накачки, заимствованные из работ: $a-[8], b-[12], c-[13]$.

\section{2. Обсуждение полученных результатов}

Проведем сравнение теоретических и экспериментальных зависимостей спектра и мощности излучения. На рис. 2 представлены экспериментальные спектральные и мощностные характеристики, заимствованные из работ $[8,12,13]$. Основной особенностью характеристик является наличие разрывов на спектральных и мощностных зависимостях, которые коррелируют друг с другом, при этом величина разрыва на ВтАХ возрастает с увеличением тока накачки ЛД.

Для расчетов принимались следующие значения величин: $\Gamma_{a}=9.6 \cdot 10^{-3}, L_{1}=0.06 \mathrm{~cm}, L_{3}=30 \mu \mathrm{m}, n_{10}=3.3$, $n_{20}=1.452, n_{3}=1, R_{1}=0.3, R_{2}=0.04, R_{3}=0.005$, $\alpha_{01}=15 \mathrm{~cm}^{-1}, \quad \alpha_{2}=0, \quad \partial n_{1} / \partial \lambda=-7 \cdot 10^{3} \mathrm{~cm}^{-1}, \quad R_{g}=$ $=5 \Omega,\left(\partial n_{1} / \partial T\right) / n_{10}=0.77 \cdot 10^{-4}, A=2 \cdot 10^{8} \mathrm{~s}^{-1}$.

Длина волны генерации ЛД с ВБР зависит от конкретного экспериментального образца решетки, длины 

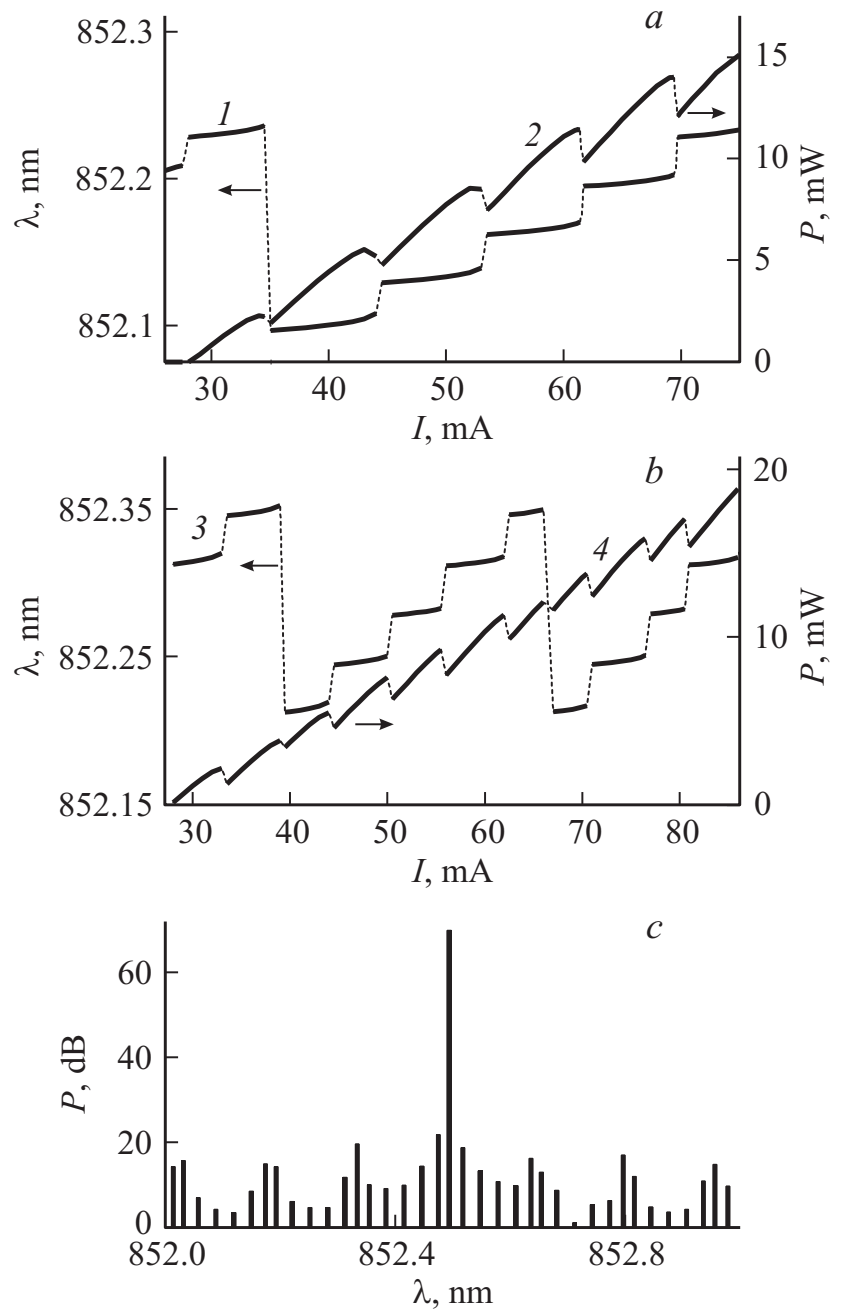

Рис. 3. Расчетные зависимости длины волны $(1,3)$ и мощности $(2,4)$ для: $a-R_{T}=55 \mathrm{~K} / \mathrm{W}, \lambda_{B}=852.16 \mathrm{~nm}$, $L_{2}=0.76 \mathrm{~cm}, b-R_{T}=82 \mathrm{~K} / \mathrm{W}, \lambda_{b}=852.28 \mathrm{~nm}, L_{2}=0.74 \mathrm{~cm}$ и $c$ - рассчитанный спектр излучения для величины тока накачки $I=50 \mathrm{~mA}, \lambda_{B}=852.5 \mathrm{~nm}, L_{2}=0.76 \mathrm{~cm}$.

световода, теплового сопротивления ЛД, показателя преломления ЛД и т. д., поэтому трудно получить полное совпадение теоретических и экспериментальных зависимостей. Будем считать, что теоретические и экспериментальные зависимости удовлетворительно совпадают друг с другом, если при увеличении тока накачки длина волны увеличивается при переключении генерации по модам внешнего резонатора и разрывы на спектральной характеристике коррелируют с разрывами на ВТАХ, а величина разрыва на ВтАХ возрастает с увеличением тока накачки.

На рис. 3, а представлены расчетные зависимости длины волны и мощности излучения от тока накачки ЛД для $R_{T}=55 \mathrm{~K} / \mathrm{W}, \lambda_{b}=852.16 \mathrm{~nm}, L_{2}=0.76 \mathrm{~cm}$. На рис. $3, b$ представлены расчетные зависимости от тока накачки ЛД для $R_{T}=82 \mathrm{~K} / \mathrm{W}, \lambda_{B}=852.28 \mathrm{~nm}, L_{2}=0.74 \mathrm{~cm}$. На рис. 3,c представлен спектр излучения для тока накачки $I=50 \mathrm{~mA}$ и $R_{T}=55 \mathrm{~K} / \mathrm{W}, \lambda_{B}=852.5 \mathrm{~nm}$, $L_{2}=0.76 \mathrm{~cm}$.

Сравнивая теоретические и экспериментальные зависимости, видим, что они удовлетворительно совпадают друг с другом.

Расчеты показывают, что основное влияние на характер поведения спектральных и мощностных характеристик оказывает учет нагрева активной области ЛД по формуле (12). Если положить величину теплового сопротивления равной нулю, то длина волны излучения не будет зависеть от тока накачки, а мощность излучения будет линейно возрастать с увеличением тока накачки. Аналогичное поведение системы будет, если пренебречь зависимостью показателя преломления активной области ЛД (17) от температуры. Если в формуле (12) исключить зависимость температуры активной области ЛД от мощности излучения, выводимой из резонатора ЛД, то будут отсутствовать разрывы на ВтАХ.

На рис. 4, $a$ представлены расчетные зависимости длины волны и мощности излучения от тока накачки для $R_{T}=40 \mathrm{~K} / \mathrm{W}, \lambda_{B}=852.16 \mathrm{~nm}, L_{2}=0.76 \mathrm{~cm}$.

На рис. $4, b$ представлены зависимость ширины линии генерации одиночного ЛД, рассчитанная по (19), и зависимость ШЛГ ЛД с ВБР, рассчитанная по (18). Для расчетов выбирались следующие величины: $n_{\mathrm{sp}}=2.6$, $\alpha_{H}=5, \alpha_{\text {end }}=L_{1}^{-1} \ln \left(R_{1}^{-1}\right), \alpha_{10}=15 \mathrm{~cm}^{-1}, R_{T}=40 \mathrm{~K} / \mathrm{W}$. При этом в диапазоне токов накачки 50-80 mA ШЛГ для ЛД с ВБР слабо зависит от тока накачки.

Расчеты показывают, что ШЛГ может быть уменьшена на четыре порядка.
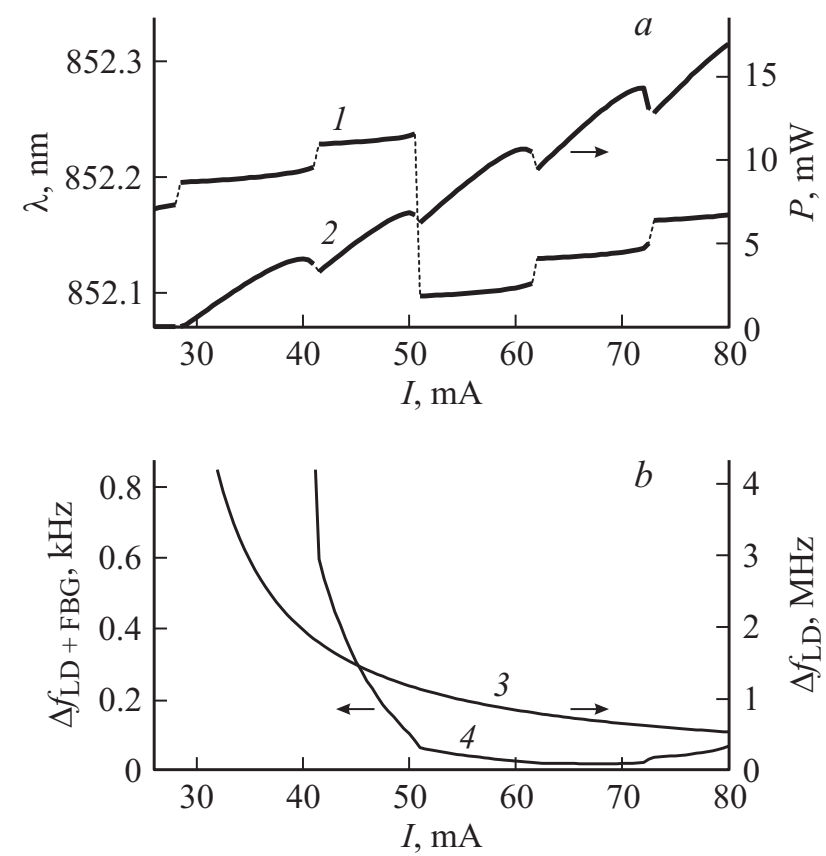

Рис. 4. Расчетные зависимости: $a$ - длины волны (1) и мощности (2) для $R_{T}=40 \mathrm{~K} / \mathrm{W}, \lambda_{B}=852.16 \mathrm{~nm}, L_{2}=0.76 \mathrm{~cm}$, $b$ - ширины линии генерации одиночного ЛД (3) и ЛД c ВБР (4). 
В работе [14] указывается, что по результатам теоретических и экспериментальных исследований для величины $\Delta f_{\mathrm{LD}} / \Delta F$ получены значения в диапазоне от $10^{3}$ до $4 \cdot 10^{4}$, где $\Delta F-$ ШЛГ для ЛД с внешней оптической обратной связью. То есть ШЛГ для ЛД с внешней оптической обратной связью может быть уменьшена более чем на четыре порядка, что коррелирует с результатами, полученными в настоящей работе.

По сообщению авторов работы [15] для ЛД длиной $400 \mu \mathrm{m}$ и расстоянию до решетки $12 \mathrm{~cm}$ измеренная ШЛГ составляла величину от 8 до $48 \mathrm{kHz}$ (для длины волны излучения $780 \mathrm{~nm})$.

Для ЛД с объемной решеткой внутренняя величина ШЛГ составляет $3.6 \mathrm{kHz}$ при мощности излучения $62 \mathrm{~mW}$ [16] и $300 \mathrm{~Hz}$ при мощности излучения $35 \mathrm{~mW}$ сообщена авторами работы [17].

\section{Заключение}

1. Расчет спектральных и мощностных характеристик по упрощенной модели позволяет получить удовлетворительное совпадение теории и эксперимента.

2. Величина ШЛГ для ЛД с ВБР может быть уменьшена на четыре порядка по сравнению с ШЛГ одиночного ЛД.

\section{Список литературы}

[1] Lang R., Kobayashi K. // IEEE J. Quant. Electron. 1980. Vol. 16. N 3. P. 347-355.

[2] Hui R.-Q., Tao S.-P. // IEEE J. Quant. Electron. 1989. Vol. 25. N 6. P. $1580-1584$.

[3] Park J.-D., Seo D.S., McInerney J.G. // IEEE J. Quant. Electron. 1990. Vol. 26. N 8. P. 1353-1362.

[4] Abdulrhmann S.G., Ahmed M., Okamoto T., Ishimori W., Yamada M. // IEEE J. Sel. Top. Quant. Electron. 2003. Vol. 9. N 5. P. $1265-1274$.

[5] Журавлева О.В., Иванов А.В., Курносов В.Д., Курносов К.В., Мустафин И.Р., Симаков В.А., Чернов Р.В., Плешанов С.А. // Квант. электрон. 2008. Т. 38. № 4. C. 319-324.

[6] Marcuse D., Lee T.-P. // IEEE J. Quant. Electron. 1984. Vol. 20. N 2. P. $166-176$.

[7] Иванов А.В., Курносов В.Д., Курносов К.В., Романцевич В.И., Чернов Р.В., Мармалюк А.А., Волков Н.А., Жолнеров В.С. // Квант. электрон. 2011. Т. 41. № 8. C. 692-696.

[8] Жолнеров В.С., Иванов А.В., Курносов В.Д., Курносов К.В., Лобиниов А.В., Романцевич В.И., Чернов Р.В. // ЖТФ. 2012. Т. 82. Вып. 6. С. 63-68.

[9] Жолнеров В.С., Иванов А.В., Курносов В.Д., Курносов К.В., Романщевич В.И., Чернов Р.В. // ЖТФ. 2014. Т. 84. Вып. 3. С. 108-112.

[10] Li L. // IEEE J. Quant. Electron. 1990. Vol. 26. N 1. P. 3-5.

[11] Henry C. // IEEE J. Quant. Electron. 1982. Vol. 18. N 2. P. 259-264.

[12] Жолнеров В.С., Иванов А.В., Курносов В.Д., Курносов К.В., Романцевич В.И., Чернов Р.В. // Квант. электрон. 2013. T. 43. № 9. С. 824-827.
[13] Журавлева О.В., Иванов А.В., Курносов В.Д., Курносов К.В., Леонович А.И., Чернов Р.В., Шишков В.В., Плешанов С.А. // Квант. электрон. 2006. Т. 36. № 8. C. 741-744.

[14] Petermann $K$. Laser diode modulation and noise. Kluwer Academic. 1988. P. 267.

[15] Genty G., Grohn A. etal. IEEE J. Quant. Electron. 2000. Vol. 36. N 10. P. 1193-1198.

[16] Luvsandamdin E., Mura G. et al. // Proc. Intern. Conf. of Space Optical System and Application. Santa Monica. CA, 2011.

[17] Bawamia A., Schimangk M. et al. // Proc. Intern. Conf. of space Optical System and Application. France, 2012. 